\title{
IDEMPOTENTS IN MATRIX RINGS
}

\author{
CHRISTOPHER BARNETT AND VICTOR CAMILLO
}

(Communicated by Eric M. Friedlander)

\begin{abstract}
Let $R$ be a commutative, von Neumann regular ring and $M_{n}(R)$ the ring of $n \times n$ matrices over $R$. What are the idempotents in $M_{n}(R)$ ? Our motivation is to think of $R$ as the sort of ring that occurs in functional analysis, for example a ring of measurable functions. We show how to uniquely write down all idempotents in $M_{n}(R)$ in terms of arbitrary parameters. The main theorem is stated in language to appeal to an audience wider than algebraists, but in a remark, we give a more refined statement for specialists.
\end{abstract}

In [1] the authors showed that every such idempotent above is conjugate to a unique diagonal matrix $D_{t}$ as described in the definition section of the text. This left open the problem of uniquely writing down the idempotents $E$ in terms of the $D_{t}$ and some set of parameters. In this paper we give a solution to that problem, which is the content of Theorem 3.

This problem was really quite messy until Gordon James provided a suggestion to handle the field (local) case and Walter Burgess told us how to use the basic ideas in Pierce's monograph [2] to globalize the local solutions. We thank both for their interest and help.

This is one of those articles whose notation could obscure some fairly simple ideas, so we have written it a bit heuristically in spots, asking forgiveness in advance for any obscurity.

If $R$ is a commutative von Neumann regular ring, then localizing at a maximal ideal $M$ is the same as dividing by that maximal ideal, so, sometimes we use these words interchangeably.

Definitions. If $0 \leq t \leq n, N$ is an $(n-t) \times t$ matrix, and $M$ is $t \times(n-t)$ matrix, then we denote the idempotent (check it) matrix

$$
\left[\begin{array}{cc}
-M N & M \\
-(N M N+N) & N M+I_{n-t}
\end{array}\right]
$$

by $S[M, N, t]$.

Let $F$ be a field, and let $D_{t} \in M_{n}(F)$ be the diagonal matrix whose first $t$ diagonal entries are 0 , and whose next $n-t$ diagonal entries are 1 .

We call a permutation $\sigma \in S_{n}$ a t-permutation if $\sigma$ preserves the order of the intervals $[1, t]$ and $[t+1, n]$, as a monomorphism into $[1, n]$. A

Received by the editors March 13, 1992.

1991 Mathematics Subject Classification. Primary 16A89, 16A49; Secondary 16A36, 16A42. 
permutation matrix is called a $t$-permutation matrix if it permutes rows as a $t$-permutation. If $N$ is an $(n-t) \times t$ matrix and $G \in S_{n}$ is a permutation, we say $G$ is $N$-admissible if the matrix obtained by letting $G$ act on the rows of the $n \times t$ matrix $\left[\begin{array}{l}I_{t} \\ N\end{array}\right]$ results in a matrix that remains in column reduced form (see Lemma 1). If $P$ is a permutation matrix, we say $P$ is $N$-admissible if $P$ acting as a permutation is $N$-admissible.

A matrix over a commutative ring is locally a t-permutation matrix if it is a $t$-permutation matrix at every prime (in this case) maximal ideal $M$.

In what follows we will have to deal with some subtleties involving how we add idempotents to get $R$. So, for convenience, we make the following definitions. Let $S=\left\{f_{1}, \ldots, f_{n}\right\}$ be a set of idempotents. We say $S$ is an ordered set if the order in which the idempotents are written down matters. $S$ is a unit set if $f_{1}+f_{2}+\cdots+f_{n}=1 . S$ is an orthogonal set if $f_{i} f_{j}=\delta_{i j}$.

We need a small observation, which we isolate as a lemma. First, a definition. If $V$ is an $n$-dimensional vector space with ordered basis $\left\{e_{1}, \ldots, e_{n}\right\}=B$ and $v \in V$ we define $h(v)$ to be the least $t$ such that the coefficient of $e_{t}$ in the representation of $v$ with respect to $B$ is not zero. We say $v$ is in unit form with respect to the basis $B$ if the $h(v)$ coefficient of $v$ is 1 .

Lemma 1. If $W$ is a subspace of a vector space $V$ and $V$ has ordered basis $B$, then $W$ has a unique ordered basis in unit form, $\omega=\left\{\omega_{1}, \omega_{2}, \ldots, \omega_{t}\right\}$ with $h\left(\omega_{1}\right)<h\left(\omega_{2}\right)<\cdots<h\left(\omega_{t}\right)$ and the $h\left(\omega_{k}\right)$ coordinates of $\omega_{i}$ are zero if $i \neq k$.

Proof. The argument is straightforward, amounting to row reduction.

Theorem 1. Let $F$ be a field and $E$ an idempotent in $M_{n}(F)$ with $\operatorname{dim}(\operatorname{Im} E)$ $=t$. Then there is a unique $t \times(n-t)$ matrix $M$, a unique $(n-t) \times t$ matrix $N$, and a unique $N$-admissible t-permutation matrix $P$ such that

$$
E=P S(M, N, t) P^{-1} .
$$

Proof. Let $Z$ denote the centralizer of $D_{t}$ in $\mathrm{GL}_{n}(F)$. Then, a simple computation shows that $Z=\left\{\left[\begin{array}{cc}G_{t} & 0 \\ 0 & G_{n-t}\end{array}\right]\right\}$, the blocked matrices where $G_{t}$ and $G_{n-t}$ are invertible $t \times t$ and $(n-t) \times(n-t)$ matrices. Now, by the usual change of basis argument, every idempotent in $M_{n}(F)$ whose image has dimension $t$ is conjugate to $D_{t}$. If $E$ is such an idempotent, with $E=U D_{t} U^{-1}=V D_{t} V$, then $V^{-1} U \in Z$, so, to find unique invertible matrices to conjugate $D_{t}$ we need to find a unique set of left coset representatives for $\operatorname{GL}_{n}(F) / Z$.

To do this, we use a suggestion of Gordon James: find left coset representatives for the group of the form $\left\{\left[\begin{array}{cc}G_{t} & M \\ 0 & G_{m-t}\end{array}\right]\right\}=U$, when $U$ is divided by $Z$, and left coset representatives for $\operatorname{GL}_{n}(F) / U$. First, $\left\{\left[\begin{array}{cc}I_{t} & M \\ 0 & I_{n-t}\end{array}\right]\right\}$ is clearly a set of coset representatives for $U / Z$. Now, if we think of $\operatorname{GL}_{n}(F)$ as written with respect to an $n$ ordered basis $\left\{e_{1}, \ldots, e_{t}, \ldots, e_{n}\right\}$, then $U$ is the subgroup of $\mathrm{GL}(n, F)$ that leaves $\sum_{k=1}^{t} e_{k} F=B_{t}$ invariant.

To find a set of left coset representatives, for each $t$-dimensional subspace $W$ of $F^{n}$, we need to find a unique invertible linear map $f$, such that $f B_{t}=W$. Then, if $g \in \mathrm{GL}_{n}(F)$ we have $f B_{t}=g B_{t}$, so $f^{-1} g$ leaves $B_{t}$ invariant, and $\{f\}$ will be the desired set of coset representatives. Now, for every such subspace $W$, we find the unique basis, $\left\{V_{i}\right\}$, for $W$ as indicated in the lemma. Write the coefficients of this basis as an $n \times t$ matrix whose $i$ th column is the 
coefficients of $V_{i}$. If we call this matrix $X$, it is clear that we can permute the rows of $X$ to put $I_{t}$ on the top. That is, there is a (not necessarily unique) permutation matrix $Q \in \mathrm{GL}_{n}(F)$, such that $Q X=\left[\begin{array}{l}I_{t} \\ N\end{array}\right]$.

To ensure uniqueness, write $X=P\left[\begin{array}{l}I_{t} \\ N\end{array}\right]$ where $P$ is an $n \times n$ permutation matrix. But, note, $P$ must be $N$-admissible since $X$ is column reduced. There is no uniqueness here, either for $Q$ or $N$. [Example,

$$
\left[\begin{array}{l}
1 \\
a \\
b
\end{array}\right]=I_{3}\left[\begin{array}{l}
1 \\
a \\
b
\end{array}\right]=P\left[\begin{array}{l}
1 \\
b \\
a
\end{array}\right]
$$

where $P$ interchanges the last two rows.] But, if we consider the form of $X$ as the coordinates in canonical form for a basis for $W$, and if we require $Q$ to be a $t$-permutation, then both $Q$ and $N$ are unique. This is because the rows in which the initial ones occur increase as the indices of the $V_{i}$ increase, and $N$ can be made by "collapsing" the other rows, without changing their order.

Now, we can embed $\left[\begin{array}{l}I_{t} \\ N\end{array}\right]$ in the invertible matrix $\left[\begin{array}{cc}I_{t} & 0 \\ N & I_{n-t}\end{array}\right]$ so we have that: $\left\{P\left[\begin{array}{cc}I_{t} & 0 \\ N & I_{n-t}\end{array}\right]\left[\begin{array}{cc}I_{t} & M \\ 0 & I_{n-t}\end{array}\right]\right\}$ is a unique set of coset representatives, and if we conjugate $D_{t}$ by these, after a straightforward calculation we get the theorem.

The following globalization argument is really the content of Pierce [2, 3.4]. We present a short proof here for convenience, and because it illustrates how the much more general ideas in Pierce's monograph work.

Theorem 2 (Richard Pierce). Let $R$ be a commutative von Neumann regular ring, and let $E$ be an idempotent in $M_{n}(R)$ which is conjugate to $D_{t}$ for some $t$. Then there exist unique matrices $M$ and $N$ and a unique locally t-permutation locally $N$-admissible matrix $P$ such that

$$
E=P F(N, M, t) P^{-1} .
$$

Proof. Let $X$ be a maximal ideal and let - denote the image $\bmod M$. Then, by Theorem 1:

(1) $\bar{E}=\bar{P} S(\bar{M}, \bar{N}, t) \bar{P}^{-1}$, where $\bar{P}$ is a unique $N$-admissible $t$-permutation matrix and $\bar{M}$ and $\bar{N}$ are unique (note the - ). Then, using the ideas of Pierce the matrix $E-P S(M, N, t) P^{-1}$ has entries that lie in $X$, and so generate an ideal generated by an idempotent $(1-e)$. Then $e \notin X$ and $e\left(E-P S(M, N, t) P^{-1}\right)=0$ so

(2) $e E=e P S(e M, e N, t) e P^{-1}$.

So, for every maximal ideal $X_{l}$, find an idempotent, which we denote by $e_{l}$, and matrices $P_{l}, M_{l}$, and $N_{l}$ satisfying (2). Since $\left\{e_{l}\right\}$ are contained in no maximal ideal, they generate the whole ring, so by renumbering we can assume $e_{1}+\cdots+e_{n}$ is a unit. Then, define $f_{l}=e_{l} \prod_{k<l}\left(1-e_{k}\right)$. The $f_{l}$ are an orthogonal unit set. Let

$$
M=\sum_{l=1}^{n} f_{l} M_{l}, \quad N=\sum_{l=1}^{n} f_{l} N_{l}, \quad P=\sum_{l=1} f_{l} P_{l} .
$$

Then we claim

(3) $E=P S(M, N, t) P^{-1}$ and $P, M, N$ are unique as described in the theorem. We need only show equality locally. Let $X$ be any maximal ideal; then exactly one of the $f_{k} \notin X$ in (1), so $f_{e} \equiv e \equiv 1 \bmod X($ since $R / X$ is a 
field and has only trivial idempotents). So, if we let - denote image $\bmod X$, we have $\bar{M}=\overline{f_{l} M_{l}}=f_{l} \bar{M}=\overline{e M}$, etc. So, if we reduce the right side of (3) $\bmod X$ we end up with $\overline{e E}=\bar{E}$. This establishes (3).

The uniqueness follows because $P, M, N$ satisfy Theorem 1 , mod any maximal ideal, and are unique locally, so they are unique globally.

Now, we know that if $E \in M_{n}(R)$ is an idempotent, then $E$ is conjugate to a unique diagonal matrix $\operatorname{diag}\left(e_{1}, \ldots, e_{n}\right)$ where $e_{i} e_{j}=e_{i}$ if $i \leq j$ [1]. If we let $e_{0}=0$ and $f_{i}=e_{i}-e_{i-1}$ then one easily computes $f_{i} f_{j}=\delta_{i j} f_{i}$.

(1) $\operatorname{diag}\left(e_{1}, \ldots, e_{n}\right)=\sum_{i=1}^{M} f_{i} D_{i}$ and $e_{n}=f_{1}+\cdots+f_{n}$ so that there is a one-to-one correspondence between matrices of the form on the right and left sides of (1) above.

Theorem 2 and the above give:

Theorem 3. Let $R$ be a commutative von Neumann regular ring. Then, there exists a unique, ordered, orthogonal unit set of idempotents $\left\{f_{1}, \ldots, f_{n}\right\}$, unique matrices $\left\{M_{t}\right\},\left\{N_{t}\right\}$, and unique locally $N_{t}$-admissible t-permutation matrices $P_{t}$, such that

$$
E=\sum_{t=1}^{n} f_{t} P_{t} S\left(M_{t}, N_{t}, t\right) P_{t}^{-1} .
$$

Remark 1. Consider the uniqueness of a locally $t$-permutation locally $N$-admissible matrix $P$. Pierce's arguments show that every such matrix lifts locally to a $t$-permutation $N$-admissible matrix, so that in the end we have $P=\sum_{k=1}^{m} f_{k} P_{k}$, where $\left\{f_{1}, \ldots, f_{n}\right\}$ is an orthogonal unit set. Over a field there are only finitely many [in fact, $\left.\left(\begin{array}{l}n \\ t\end{array}\right)\right] t$-permutation matrices, so we can collect the $f$ 's together to multiply these and still get an orthogonal spanning set. So, if

$$
P=\sum_{k=1}^{\left(\begin{array}{c}
n \\
t
\end{array}\right)} f_{k} P_{k}=\sum_{k=1}^{\left(\begin{array}{c}
n \\
t
\end{array}\right)} g_{k} P_{k},
$$

consider an $f_{s}$, then there is at least one $t$ such that $f_{s} g_{t} \neq 0$ since $R=\sum g_{t} R$, so that $f_{s} g_{t} P_{s}=f_{s} g_{t} P_{t}$. This clearly means $s=t$, since the $P$ 's are $\{0,1\}$ matrices. Then $f_{s} g_{t}=0$ if $s \neq t$ so since $\sum g_{t}=1$ we have $f_{s}=g_{s}$.

Remark 2. The following is a formulation of Theorem 3 for algebraists in the form suggested by Walter Burgess, as indicated in the introduction.

Theorem. Given $E=E^{2} \in M_{n}(R)$ there is a unique orthogonal unit set $\left\{e_{1}, \ldots\right.$, $\left.e_{n}\right\}$ in $R$ so that for $x \in \operatorname{supp} e_{t}, \operatorname{rank}\left(e_{t}\right)_{x}=t$. For each $t>0$ with $e_{t} \neq 0$ there are unique matrices with entries from $R e_{t}, M_{t} \in M_{t, n-t}\left(e_{t} R\right)$, $N_{t} \in M_{n-t, t}\left(e_{t} R\right)$, and $P_{t} \in M_{n}\left(e_{t} R\right)$ so that $P_{t}$ is locally an $N_{t}$-admissible $t$-permutation matrix on supp $e_{t}$, such that

$$
e_{t} E=P_{t} S\left(M_{t}, N_{t}, t\right) P_{t}^{-1} \in M_{n}\left(e_{t} R\right) .
$$

Moreover, for each $t>0$ so that $e_{t} \neq 0$, there is a unique orthogonal unit set of idempotents $q_{t_{1}}, \ldots, q_{t_{m}}$ for $e_{t}$ so that for $j=1, \ldots, m, q_{t_{j}} P_{t}$ is a $t$-permutation matrix in $M_{n}\left(q_{t_{j}} R\right)$. This means that given an idempotent $E$ we can simply write $R=\prod_{i=1}^{n} R_{i}$ as a product of rings so that $E$ has rank $t$ at every maximal ideal in $R_{t}$. Further, each $R_{t}$ can be decomposed into a 
finite number of factors so that the locally $t$-permutation matrices that occur are actually $t$-permutation matrices on these factors.

\section{REFERENCES}

1. C. Barnett and V. Camillo, Idempotents in matrices over commutative von Neumann regular Rings, Comm. Algebra 18 (1990), 3905-3911.

2. R. S. Pierce, Modules over commutative von Neumann regular rings, Mem. Amer. Math. Soc., vol. 70, Amer. Math. Soc., Providence, RI, 1967.

3. K. R. Goodearl, Von Neumann regular rings, Pitman, New York, 1978.

Department of MAThematics, Imperial College, London, England

Department of Mathematics, The University of Iowa, Iowa City, Iowa 52242 